\title{
Inguinal hernia treatment with the Prolene Hernia System in a Dutch regional training hospital
}

\author{
C. A. S. Berende $\cdot$ J. P. Ruurda $\cdot$ C. E. V. B. Hazenberg $\cdot$ \\ J. G. Olsman • H. J. A. A. van Geffen
}

Received: 13 November 2006 / Accepted: 26 February 2007 / Published online: 4 April 2007

(C) Springer-Verlag 2007

\begin{abstract}
Introduction In this retrospective study results from inguinal hernia repair with the Prolene Hernia System (PHS) in a regional training hospital were analysed.

Patients and methods One-hundred and seventy-eight primary inguinal hernias and thirty-nine recurrent hernias (initial non-mesh repair) were treated with the PHS. The primary endpoint was the recurrence rate. Secondary endpoints were short-term and long-term complications. Pain was evaluated by use of a visual analog scale (VAS, 0-100), and a short-form 36-item questionnaire was used to assess postoperation quality of life. All patients visited the outpatient clinic for a physical examination $(100 \%$ follow-up).

Results After a median follow-up of 32 months four patients were diagnosed with recurrent herniation (1.8\%), three after primary hernia repair $(1.6 \%)$ and one after recurrent hernia repair $(2.6 \%)$. Three superficial wound infections $(1.3 \%)$, three haematomas needing surgical evacuation $(1.3 \%)$, and two lesions of the spermatic cord $(0.9 \%)$ were diagnosed. Seven patients $(3.2 \%)$ suffered from persistent pain (VAS $>40$ ). Average VAS score was $13(0-80)>24$ months after surgery.

Conclusion In a regional training hospital, primary and recurrent inguinal hernias were treated with low recurrence and few complications by use of the PHS.
\end{abstract}

Keywords Inguinal hernia $\cdot$ Bilayer device . Myopectineal orifice $\cdot$ Recurrence $\cdot$ Pain

C. A. S. Berende - J. P. Ruurda · C. E. V. B. Hazenberg

J. G. Olsman · H. J. A. A. van Geffen ( $\bowtie)$

Department of Surgery, Jeroen Bosch Hospital,

Nieuwstraat 34, 5211 's-Hertogenbosch, The Netherlands

e-mail: E.v.Geffen@jbz.nl

\section{Introduction}

Because inguinal hernia repair is the most frequently performed procedure in general surgery, a small decrease in the incidence of recurrence, re-operation, and morbidity will have great socio-economic consequences and will, therefore, affect the choice of technique [1].

After the introduction of tension-free surgical repair with use of prosthetic mesh, recurrence and patient comfort improved substantially compared with use of traditional, tension-producing techniques [2-4]. The optimum operating technique for groin hernia has still not been established, however. Several types of prosthesis have been developed and used in different positions to reduce the number of recurrences [5-8]. Pre-peritoneal placement of mesh, posterior to the inguinal canal, seems the most logical choice mechanically [9]. For some techniques using a pre-peritoneal approach, for example totally extraperitoneal hernioplasty, there is a long learning curve. Laparoscopic techniques are also more expensive, operating times are longer, and when complications occur they tend to be more severe than for an anterior approach [10].

A concept combining an anterior approach and posterior mesh augmentation of the inguinal canal was introduced in 1998. Previous reports analysing results from inguinal hernia treatment with the bilayer Prolene Hernia System (PHS; Ethicon, Norderstedt, Germany) have revealed that recurrence and morbidity are low $[1,11,12]$. Most of these reports were from specialized hernia clinics and the role of inguinal hernia repair with this device in a teaching hospital is still unclear.

The objective of this study was to retrospectively analyse results from inguinal hernia treatment with the PHS in a regional training hospital. 


\section{Patients and methods}

Patients over 18 years of age scheduled for elective inguinal hernia repair in our clinic using the PHS during the period 2002-2004 were included in this study. Both primary and recurrent (initial non-mesh repair) inguinal hernias were included. Patients were excluded if the hernia was strangulated, if they had previous mesh repair, or if they were ASA class IV or V [13]. No additional patient selection was performed.

All patients visited the outpatient clinic 24 months or more postoperatively for a physical examination. If persistent pain was being experienced and/or there was clinical suspicion of recurrence, a qualified radiologist performed an ultrasound investigation.

The primary concern in the study was recurrence of hernia within at least 2 years after the PHS repair. Secondary concerns were short-term and long-term complications and death. Short-term complications included intra-operative recognized damage to vital structures, haematoma (needing surgical re-exploration), and wound infection, defined by Centre of Disease Control [14].

Long-term complications were assessed including pain, hypaesthesia, and testicular atrophy.

Persistent pain was defined as pain on a visual analog scale $($ VAS $)>40 / 100$ at least 24 months after surgery [15]. The innervation zones of the ilioinguinal, genitofemoral, and iliohypogastric nerve were also examined physically for areas of pinprick hypaesthesia. Hypaesthesia was defined as sensory change, sensory loss, or numbness in those subsequent innervation areas compared with normal skin. The Prader orchidometer was used to assess testicular volume.

Patients completed a short form (SF) 36-item questionnaire; the results obtained were transformed into 100-point scales for eight dimensions of well-being [16].

\section{Surgeons and surgical technique}

General surgeons with different specialties and levels of expertise in hernia surgery, and surgical residents (postgraduate years 1-6), performed the operations. Regional or general anaesthesia was used. In all procedures an oblique 4-7 cm incision was made overlying the inguinal canal. The external oblique aponeurosis was opened and, for indirect hernias, blunt dissection was used to separate the sac from the cord. The hernia sac was ligated and resected. For direct types the transversalis fascia was opened and blunt dissection of the posterior space was performed. For indirect types the internal inguinal ring was used for entry to the preperitoneal space. Inferior epigastric vessels were not routinely ligated. The PHS was placed with the underlay covering the entire myopectinal orifice (MPO), the onlay extracted, fixed around the spermatic cord with Vicryl (Ethicon) and fixed medial with Vicryl (Prolene during the first 6 months; Ethicon). Paracetamol or non-steroid antiinflammatory drugs were recommended for control of postoperation pain. Physical activity after the operation was not restricted unless such activity caused pain.

\section{Results}

From January 2002 to January 2004193 patients were treated for inguinal hernia with the PHS. In 30 patients bilateral hernias were treated, and $39(20 \%)$ were recurrent hernias. Six patients died during follow-up because of cardiovascular causes, more than 30 days after surgery.

All patients visited the outpatient clinic or were visited at their private address for follow-up more than 24 months postoperation (100\% follow-up).

Patient and hernia characteristics are listed in Tables 1 and 2 .

The mean duration of the operation was 34 (15-90) min. General anaesthesia was provided to 120 patients and 67 received regional anaesthesia. In 51 out of 187 patients (27\%) a surgical resident performed the procedure.

During the period of implementation of this technique in our hospital the medium-size PHS was used initially but the large-size PHS gradually became the standard device. A total of 63 medium-size and 154 large-size PHS meshes were used.

Table 1 Patient characteristics

\begin{tabular}{lll}
\hline Total $(n=187)$ & Primary $(n=148)$ & Recurrent $(n=39)$ \\
\hline Mean age (years) & $62(28-92)$ & $63(28-84)$ \\
Sex ratio (M:F) & $138: 10$ & $37: 2$ \\
\hline
\end{tabular}

Table 2 Hernia characteristics

\begin{tabular}{lll}
\hline Total $(n=217)$ & Primary $(n=178)$ & Recurrent $(n=39)$ \\
\hline Right sided & 75 & 14 \\
Left sided & 61 & 7 \\
Bilateral & 21 & 9 \\
Nyhus' classification [17] & \\
I & 0 & 0 \\
II & 20 & 0 \\
IIIa & 84 & 0 \\
IIIb & 74 & 0 \\
IV & 0 & 39 \\
Operation time (min) & $28(15-80)$ & $38(20-90)$ \\
\hline
\end{tabular}


After a median follow-up of 33 (range 24-50) months, three recurrences $(1.6 \%)$ were diagnosed in the primary inguinal hernia repair group and one $(2.6 \%)$ in the recurrent hernia group.

Short-term complications included two instances of iatrogenic damage to the spermatic cord $(0.9 \%)$. In three cases re-exploration for haematoma was necessary $(1.4 \%)$ and wound infection occurred in three patients (1.4\%). All infections were superficial and no mesh removal was necessary.

One patient had testicular atrophy after PHS repair of his third non-mesh recurrence. According to our questionnaire seven patients $(3.2 \%)$ experienced discomfort from hypaesthesia but none of the patients was aware of foreign body sensations. Our meticulous physical examination, however, revealed symptoms of hypaesthesia in 19 patients $(8.8 \%)$. Persistent pain (VAS > 40) was found in $3.2 \%$ of all cases. In six cases the pain was located medially, at the pubic bone. No relationship between pain and hypaesthesia was found. The mean VAS pain score after 24 months was 13 (0-80).

The SF 36-item questionnaire revealed no statistically significant difference from scores for typical Dutch inhabitants [18]; this questionnaire was also scored after 24 months. (Table 3)

\section{Discussion}

In this retrospective study results from treatment of inguinal hernia with the PHS in a regional training hospital were analysed. All 187 patients visited the outpatient clinic or were visited at their private address for a physical examination to accomplish $100 \%$ follow-up. Recurrence of $1.6 \%$ for primary hernia and $2.6 \%$ for recurrent hernia after PHS repair is at least comparable with results for other open

Table 3 Quality of life assessed by use of a short-form (SF) 36-item questionnaire $>24$ months after PHS hernia repair, and for typical Dutch inhabitants [18]

\begin{tabular}{lll}
\hline SF-36 & PHS repair & Typical Dutch inhabitants \\
\hline GH & 68 & 67 \\
BP & 64 & 78 \\
VT & 57 & 62 \\
MH & 63 & 71 \\
RE & 64 & 70 \\
RP & 76 & 78 \\
SF & 80 & 83 \\
PF & 72 & 75 \\
\hline
\end{tabular}

GH, general health; BP, bodily pain; VT, vitality; MH, mental health; $\mathrm{RE}$, role emotional; RP, role physical; SF, social functioning; PF, physical functioning tension-free techniques, which have been reported to be as low as $0.5 \%$ in specialized centres [19] and to vary from 1.0 to $4.9 \%$ in the hands of general surgeons using the Lichtenstein method [3, 20, 21]. Recurrence and complications after use of the Lichtenstein procedure in training hospitals have been shown to be slightly worse than in non-training hospitals, although the difference is not statistically significant [21, 22].

Earlier reports on the PHS technique from specialized hernia clinics and surgeons dedicated to hernia surgery showed recurrence was even lower $(<0.1 \%)$ [11, 12]. When the recurrences in our study were analysed in more detail it was noted that all patients who developed a recurrence were actually operated on by residents in their first or second postgraduate year, supervised by a surgeon. Recurrences occurred for three primary hernias (two type IIIa and one type IIIb) and one recurrent hernia (type IV). The period investigated also included the period of implementation of the PHS device in our hospital, so both the different levels of expertise and the learning curve might contribute to this difference.

It was, moreover, remarkable to see that all recurrences occurred after use of the medium-size PHS. This might suggest this size was insufficient to cover the entire MPO in an adult male [12]. Because the underlay patch for PHSmedium is $7 \mathrm{~cm}$ in diameter and the mean MPO surface has previously been found to be $6-8 \mathrm{~cm}$, might this be an explanation [9, 23]. This cannot be proven by our results, because of the small number of patients and the fact that the choice of medium or large-size PHS was strictly random. The large PHS device, with the 10-cm underlay, is large enough to provide sufficient coverage of the entire MPO, however.

In none of the recurrences was a herniotomy performed at surgery. In all four cases the hernial sac was repositioned (inverted), theoretically facilitating recurrent herniation.

According to the literature, overall occurrence of persistent pain varies between 1 and 37\% [24-26] which is comparable with this series. A possible explanation of the medially located pain might be the manner in which the mesh was fixed. According to records of the operations, all patients with persisting pain had been operated on during the period of implementation of the PHS in our hospital and the mesh had been fixed with a Prolene suture. After we had switched to Vicryl no further patients reported persistent pain in this series.

Hypaesthesia was diagnosed in $8.8 \%$ (19) of all patients, which is rather high according to the literature (2-4\%) [24, 25]. This figure was established after subjecting the patients to a meticulous neurological examination, however. Patients were aware of sensory loss in $3.2 \%$ of cases only.

In conclusion, PHS hernia repair has been shown to be an appropriate technique for both primary and recurrent 
inguinal hernias in a regional training hospital for surgeons with different levels of expertise. Occurrence of complications is acceptable and the patients' quality of life is not impaired after inguinal hernia repair with the PHS.

In our opinion the medium-size PHS has only a small range of safe use in adult males. Fixing of the PHS device with non-resorbable sutures should also be avoided.

\section{References}

1. Vironen J, Nieminen J, Eklund A, Paavolainen P (2006) Randomized clinical trial of Lichtenstein patch or Prolene Hernia System for inguinal hernia repair. Br J Surg 93:33-39

2. Condon RE, Nyhus LM (1995) Complications of groin hernia. In: Nyhus LM, Condon RE (eds) Hernia. 4th edn. J.B. Lippincott, Philadelphia, pp 269-282

3. Amid PK, Shulman AG, Lichtenstein IL (1996) Open "tensionfree" repair of inguinal hernias: the Lichtenstein technique. Eur J Surg 162:447-453

4. Scott NW, McCormack K, Graham P, Go PMNYH, Ross SJ, Grant AM (2002) Open mesh versus non-mesh repair of inguinal hernia. Cochrane Database Syst Rev 4:CD002197

5. Lange DH de, Aufenacker ThJ, Roest M, Simmermacher RK, Gouma DJ, Simons MP (2005) Inguinal hernia surgery in The Netherlands: a baseline study before the introduction of the Dutch guidelines. Hernia 9:172-177

6. Pikoulis E, Tsigris C, Diamantis T, Delis S, Tsasoulis P, Georgopoulos S, Pavlakis E, Leppaniemi AK, Bastounis E, Mantonakis S (2002) Laparoscopic preperitoneal mesh repair or tension-free mesh plug technique? A prospective study of 471 patients with 543 inguinal hernias. Eur J Surg 168:587-591

7. EU Hernia Trialist Collaboration (2000) Laparoscopic compared with open methods of groin hernia repair: systematic review of randomized controlled trials. Br J Surg 87:860-867

8. EU Hernia Trialist Collaboration (2000) Mesh compared with non-mesh methods of open groin hernia repair: systematic review of randomized controlled trials. Br J Surg 87:854-859

9. Wolloscheck T, Gaumann A, Heintz A, Junginger Th, Konerding MA (2004) Inguinal hernia: measurement of the biomechanics of the lower abdominal wall and the inguinal canal. Hernia 8:233241

10. Neumayer L, Giobbie-Hurder A, Jonasson O, Fitzgibbons R, Dunlop D, Gibbs J, Reda D, Henderson W (2004) Open mesh versus laparoscopic mesh repair of inguinal hernia. N Engl J Med 350:1819-1827
11. Gilbert AI, Young J, Graham MF, Divilio T (2004) Combined anterior and posterior inguinal hernia repair: intermediate recurrence rate with three groups of surgeons. Hernia 8:203-207

12. Gilbert AI, Graham MF, Voigt WJ (1999) A bilayer patch device for inguinal hernia repair. Hernia 3:161-166

13. American Society of Anesthesiologists (1963) New classification of physical status. Anesthesiology 196;24:111

14. Horan TC, Gaynes RP, Martone WJ, Jarvis WR, Emori TG (1992) CDC definitions of nosocomial surgical site infections,1992: a modification of CDC definitions of surgical wound infections. Infect Control Hosp Epidemiol 13:606-608

15. Acute Pain Management Guideline Panel (1992) Acute pain management: operative or medical procedures and trauma. Clinical practice guideline. Agency for Health Care Policy and Research, Rockville, February AHCPR publication no. 92-0032

16. Ware JE Jr, Sherbourne CD (1992) The MOS 36-item short-form health survey (SF-36). I. Conceptual framework and item selection. Med Care 30:473-483

17. Nyhus LM, Klein MS, Rogers FB, Kowalczyk S (1990) Inguinal hernia repairs. Types, patient care. AORN J 52(2):292-304

18. Hoeymans N, Lindert H, Westert GP (2005) The health status of the Dutch population as assessed by the EQ-6D. Qual Life Res 14:655-663

19. Stephenson BM (2003) Complications of open groin hernia repairs. Surg Clin North Am 83:1255-1278

20. Aufenacker TJ, de Lange DH, Burg MD, Kuiken BW, Hensen EF, Schoots IG, Gouma DJ, Simons MP (2005) Hernia surgery changes in the Amsterdam region 1994-2001: decrease in operations for recurrent hernia. Hernia 9(1):1-2

21. Nienhuijs S, Oort I, Keemers M, Strobbe L, Rosman C (2005) Randomized clinical trial comparing Prolene Hernia System, mesh plug and Lichtenstein method for open inguinal hernia repair. Br J Surg 92:33-38

22. Sondenaa K, Nesvik I, Breivik K, Korner H (2001) Long-term follow-up op 1059 consecutive primary and recurrent inguinal hernias is a teaching hospital. Eur J Surg 167:125-129

23. Pelissier EP (2001) Inguinal hernia: the size of the mesh. Hernia 5(4):169-171

24. Bay-Nielsen M, Perkins F, Kehlet H (2001) Pain and functional impairment 1 year after inguinal herniorrhaphy: a nationwide questionnaire study. Ann Surg 233(1):1-7

25. Aasvang EK, Bay-Nielsen M, Kehlet H (2006) Pain and functional impairment 6 years after inguinal herniorrhaphy. Hernia 10:316321

26. Amid PK (2004) Causes, prevention, and surgical treatment of postherniorrhaphy neuropathic inguinodynia: triple neurectomy with proximal end implantation. Hernia 8:343-349 